\title{
Homogeneous Field and WKB Approximation in Deformed Quantum Mechanics with Minimal Length
}

\author{
Jun Tao, ${ }^{1}$ Peng Wang, ${ }^{1}$ and Haitang Yang ${ }^{1,2}$ \\ ${ }^{1}$ Center for Theoretical Physics, College of Physical Science and Technology, Sichuan University, Chengdu 610064, China \\ ${ }^{2}$ Kavli Institute for Theoretical Physics China (KITPC), Chinese Academy of Sciences, Beijing 100080, China \\ Correspondence should be addressed to Peng Wang; pengw@scu.edu.cn
}

Received 19 May 2015; Revised 18 August 2015; Accepted 31 August 2015

Academic Editor: Piero Nicolini

Copyright (c) 2015 Jun Tao et al. This is an open access article distributed under the Creative Commons Attribution License, which permits unrestricted use, distribution, and reproduction in any medium, provided the original work is properly cited. The publication of this article was funded by $\mathrm{SCOAP}^{3}$.

\begin{abstract}
In the framework of the deformed quantum mechanics with a minimal length, we consider the motion of a nonrelativistic particle in a homogeneous external field. We find the integral representation for the physically acceptable wave function in the position representation. Using the method of steepest descent, we obtain the asymptotic expansions of the wave function at large positive and negative arguments. We then employ the leading asymptotic expressions to derive the WKB connection formula, which proceeds from classically forbidden region to classically allowed one through a turning point. By the WKB connection formula, we prove the Bohr-Sommerfeld quantization rule up to $\mathcal{G}\left(\beta^{2}\right)$. We also show that if the slope of the potential at a turning point is too steep, the $\mathrm{WKB}$ connection formula is no longer valid around the turning point. The effects of the minimal length on the classical motions are investigated using the Hamilton-Jacobi method. We also use the Bohr-Sommerfeld quantization to study statistical physics in deformed spaces with the minimal length.
\end{abstract}

\section{Introduction}

One of the predictions shared by various quantum theories of gravity is the existence of a minimal observable length. For example, this fundamental minimal length scale could arise in the framework of the string theory [1-3]. For a review of a minimal length in quantum gravity, see [4]. Some realizations of the minimal length from various scenarios have been proposed. Specifically, one of the most popular models is the generalized uncertainty principle (GUP) [5, 6], derived from the modified fundamental commutation relation

$$
[X, P]=i \hbar\left(1+\beta P^{2}\right)
$$

where $\beta=\beta_{0} \ell_{p}^{2} / \hbar^{2}=\beta_{0} / c^{2} m_{p l}^{2}, m_{p l}$ is the Planck mass, $\ell_{p}$ is the Planck length, and $\beta_{0}$ is a dimensionless parameter. For a review of the GUP, see [7]. With this generalization, one can easily derive the generalized uncertainty principle (GUP)

$$
\Delta X \Delta P \geq \frac{\hbar}{2}\left[1+\beta(\Delta P)^{2}\right] .
$$

This in turn gives the minimal measurable length

$$
\Delta X \geq \Delta_{\min }=\hbar \sqrt{\beta}=\sqrt{\beta_{0}} \ell_{p} .
$$

Equation (1) is the simplest model where only the minimal uncertainty in position is taken into account, while the momentum can be infinite. When incorporating the GUP into quantum field theory, one needs to generalize deformed commutation relations to include time. However, the existence of the minimal length could lead to Planck scale departures from Lorentz symmetry. Therefore, the corresponding deformed commutation relations are not Lorentz invariant and give rise to some version of the doubly special relativity [8-11]. 
In this paper we consider one-dimensional nonrelativistic quantum mechanics with the deformed commutation relation (1). To implement the deformed commutators (1), one defines $[6,12]$

$$
\begin{aligned}
& X=X_{0}, \\
& P=P_{0}\left(1+\frac{\beta}{3} P_{0}^{2}\right),
\end{aligned}
$$

where $\left[X_{0}, P_{0}\right]=i \hbar$, the usual canonical operators. One can easily show that to the first-order of $\beta,(1)$ is guaranteed. Henceforth, terms of $\mathcal{O}\left(\beta^{2}\right)$ and higher are neglected in the remainder of the paper. For a quantum system described by

$$
H=\frac{P^{2}}{2 m}+V(X),
$$

the Hamiltonians can be written as

$$
H=H_{0}+H_{1}+\mathcal{O}\left(\beta^{2}\right),
$$

where $H_{0}=P_{0}^{2} / 2 m+V\left(X_{0}\right)$ and $H_{1}=(2 \beta / 3) P_{0}^{2}$. Furthermore, one can adopt the momentum representation

$$
\begin{aligned}
& X_{0}=i \hbar \frac{\partial}{\partial p}, \\
& P_{0}=p
\end{aligned}
$$

or the position representation

$$
\begin{aligned}
& X_{0}=x \\
& P_{0}=\frac{\hbar}{i} \frac{\partial}{\partial x} .
\end{aligned}
$$

The momentum representation is very handy in the discussions of certain problems, such as the harmonic oscillator [13], the Coulomb potential $[14,15]$, and the gravitational well [16-18]. Recently, a wide class of problems, like scattering from a barrier or a particle in a square well [19-21], are discussed in position representation. Moreover, in the position representation, it is much easier to derive and discuss WKB approximation in the deformed quantum mechanics analogous to that in the ordinary quantum mechanics [22]. Thus, we adopt the position representation in this paper. In the position representation, the deformed stationary Schrodinger equation is

$$
\frac{d^{2} \psi(x)}{d x^{2}}-\ell_{\beta}^{2} \frac{d^{4} \psi(x)}{d x^{4}}+\frac{2 m(E-V(x))}{\hbar^{2}} \psi(x)=0,
$$

where we define $\ell_{\beta}^{2}=(2 / 3) \hbar^{2} \beta$ for later convenience.

Although, the homogeneous field potential $V(X)=F X$ is not studied so intensively as the quantum well, it has an important application in theoretical physics. In the ordinary quantum mechanics, the solutions to the Schrodinger equation with the linear potential are Airy functions, which are essential to derive the WKB connection formulas through a turning point. This motivates us to study the linear potential in the deformed quantum mechanics.
In the deformed quantum mechanics with minimal length, the WKB approximation formulas are obtained in [22]. In addition, the deformed Bohr-Sommerfeld quantization is used to acquire energy spectra of bound states in various potentials $[15,21-24]$. Therefore, it is interesting to derive the $\mathrm{WKB}$ connection formulas through a turning point and rigorously verify the Bohr-Sommerfeld quantization rule claimed before, which are presented in our paper. Besides, we find that if the slope of the potential is too steep at a turning point the WKB connection algorithm fails around the turning point. This is not unexpected because if one makes linear approximation to the potential around such a turning point for asymptotic matching the corrections to the wave functions due to Hamiltonian $H_{1}$ become dominant before one reaches the $\mathrm{WKB}$ valid region.

This paper is organized as follows: in Section 2 we give the integral representation of the physically acceptable wave function of the homogeneous field and its leading asymptotic behavior at large positive value of $\rho$. In Section 3, we obtain the asymptotic expansions of the physically acceptable wave function at both large positive and large negative values of $\rho$. Section 4 is devoted to deriving the WKB connection formula and the related discussions and applications. In Section 5, we offer a summary and conclusion.

\section{Deformed Schrodinger Equation}

Let us consider one-dimensional motion of a particle in a homogenous field, specifically in a field with the potential $V(X)=F X$. Here we take the direction of the force along the axis of $-x$ and let $F$ be the force exerted on the particle in the field. As discussed in Section 1, the deformed Schrodinger equation for this scenario is

$$
\frac{d^{2} \psi(x)}{d x^{2}}-\ell_{\beta}^{2} \frac{d^{4} \psi(x)}{d x^{4}}+\frac{2 m(E-F x)}{\hbar^{2}} \psi(x)=0 .
$$

In order to solve (10), a new dimensionless variable $\rho$ is introduced as

$$
\rho=\left(x-\frac{E}{F}\right)\left(\frac{2 m F}{\hbar^{2}}\right)^{1 / 3} .
$$

Equation (10) then becomes

$$
-\alpha^{2} \psi^{(4)}+\psi^{\prime \prime}-\rho \psi=0
$$

where we define another dimensionless variable $\alpha^{2}=$ $\ell_{\beta}^{2}\left(2 m F / \hbar^{2}\right)^{2 / 3}$ and the derivatives are in terms of the new variable $\rho$. The linear differential equation (12) is quartic and then there are four linearly independent solutions. We will shortly show that only one of them is physically acceptable.

2.1. Physically Acceptable Solution. The condition $\beta P^{2} \ll 1$ validating our effective GUP model implies

$$
\beta\left\langle x\left|P^{2}\right| \psi\right\rangle \ll\langle x \mid \psi\rangle \Longrightarrow \alpha^{2}\left|\psi^{\prime \prime}(\rho)\right| \ll|\psi(\rho)| .
$$

This condition is also expected in the momentum space. Since the GUP model is only valid below the energy scale $\beta^{-1 / 2}$, 
the momentum spectrum of the state $|\psi\rangle$ should be greatly suppressed around the scale $\beta^{-1 / 2}$. It also leads to condition (13). Moreover, conditions (13) and (12) give

$$
\alpha^{2}|\rho| \ll 1
$$

In other words, our GUP model, which is an effective model, is valid only when condition (14) holds. Considering that the Compton wavelength of a particle should be much larger than $\hbar \sqrt{\beta}$ or $\ell_{\beta}$ in the GUP model, one can also obtain condition (14) in the classical allowed region where $\rho<0$. In a field with the potential $V(x)$, the kinematics energy of a nonrelativistic particle is $E-V(x)$ and its momentum is $\sqrt{2 m(E-V(x))}$. Therefore, the fact that the Compton wavelength of the particle $\lambda_{c}=\hbar / \sqrt{2 m(E-V(x))}$ is much larger than $\ell_{\beta}$ yields $\alpha^{2}|\rho| \ll 1$. In the remainder of our paper except Section 4.2.1, we assume $\alpha \ll 1$ which is useful to derive WKB connection formula around a smooth tuning point. One needs to consider $\alpha \gtrsim 1$ scenario only when it comes to the WKB connection around a sharp turning point.

We notice that $E<V$ for $\rho>0$. The wave function $\psi$ is then exponentially damped for large positive value of $\rho$. Thus, one needs to evaluate asymptotic values of $\psi(\rho)$ at large positive value of $\rho$ to find physically acceptable solution to (12). Note that, only when $\alpha \ll 1$, one can analyze asymptotic behavior of $\psi(\rho)$ at large positive value of $\rho$ in the physically acceptable region where $\alpha^{2}|\rho| \ll 1$. To determine the leading behavior of $\psi(\rho)$ at large positive value of $\rho$, we make the exponential substitution $\psi(\rho)=e^{s(\rho)}$ and then obtain for (12)

$$
\begin{aligned}
s^{\prime \prime} & +s^{\prime 2}-\rho-\alpha^{2}\left[s^{(4)}+6 s^{\prime 2} s^{\prime \prime}+3 s^{\prime \prime 2}+4 s^{\prime} s^{(3)}+s^{\prime 4}\right] \\
& =0 .
\end{aligned}
$$

Equation (15) is as difficult to solve as (12). Here our strategy to find the asymptotic behavior of $\psi(\rho)$ from (15) is as follows [25]:

(a) We neglect all terms appearing small and approximate the exact differential equation with the asymptotic one.

(b) We solve the resulting equation and check that the solution is consistent with approximations made in step (a).

It is usually true that higher derivative terms than $s^{\prime}$ are discarded in step (a). Therefore, we reduce (15) to the asymptotic differential equation

$$
s^{\prime 2}-\alpha^{2} s^{\prime 4} \sim \rho .
$$

Solving (16) gives four solutions for $s^{\prime}$, two of which are discarded considering $\beta P^{2} \ll 1$. Taking asymptotic relation (13) into account, one can further reduce the quartic equation (16) to a quadratic equation

$$
s^{\prime 2} \sim \rho,
$$

which has only two solutions for $s^{\prime}$. The two solutions are $s^{\prime} \sim$ $\pm \sqrt{\rho}$ and, therefore,

$$
\psi(\rho) \sim \exp \left( \pm \frac{2}{3} \rho^{3 / 2}\right)
$$

at large positive value of $\rho$,

where - is for the physically acceptable solution. It is easy to check that the solution $s^{\prime} \sim \pm \sqrt{\rho}$ satisfies the assumptions

$$
s^{\prime \prime}, s^{\prime 2} s^{\prime \prime}, s^{(3)}, s^{\prime \prime 2}, s^{\prime} s^{(3)}, s^{(4)} \ll \rho,
$$

as long as $\rho \gg 1$.

It is interesting to note that the two discarded solutions of (16) are

$$
s^{\prime} \sim \pm \frac{\sqrt{1+\sqrt{1-4 \alpha^{2} \rho}}}{\sqrt{2 \alpha}}
$$

which become $s^{\prime} \sim \pm 1 / \sqrt{\alpha}$ when $\alpha^{2} \rho \ll 1$. The resulting wave functions are $\psi(\rho) \sim \exp ( \pm \rho / \sqrt{\alpha})$. They are not physical states since they fail to satisfy condition (13). One can also see that these two solutions are discarded according to the low-momentum consistency condition in [26]. In summary, assuming $\alpha \ll 1$, we find that the leading asymptotic behavior of the physically acceptable solution is $\exp \left(-(2 / 3) \rho^{3 / 2}\right)$ for $\rho \gg 1$. In addition, we only analyze the solution in the region $\alpha^{2}|\rho| \ll 1$, where the GUP model is valid.

2.2. Integral Representation. The differential equation (12) can be solved by Laplace's method. Please refer to mathematical appendices of [27] for more details. Define the polynomials

$$
\begin{aligned}
& P(t)=-\alpha^{2} t^{4}+t^{2} \\
& Q(t)=-1
\end{aligned}
$$

and the function

$$
\begin{aligned}
Z(t) & =\frac{1}{Q(t)} \exp \left(\int \frac{P(t)}{Q(t)} d t\right) \\
& =-\exp \left(\frac{\alpha^{2} t^{5}}{5}-\frac{t^{3}}{3}\right) .
\end{aligned}
$$

Integral representations of the solutions to (12) are then given by

$$
\begin{aligned}
\psi(\rho) & =-\int_{C} \exp (\rho t) Z(t) d t \\
& =\int_{C} \exp \left(\rho t+\frac{\alpha^{2} t^{5}}{5}-\frac{t^{3}}{3}\right) d t,
\end{aligned}
$$

where the contour $C$ is chosen so that the integral is finite and nonzero and the function

$$
V(t)=\exp \left(\rho t+\frac{\alpha^{2} t^{5}}{5}-\frac{t^{3}}{3}\right)
$$


vanishes at endpoints of $C$ since the integrand of (23) is entire on the complex plane of $t$. Now that $\exp \left(x t+\alpha^{2} t^{5} / 5-t^{3} / 3\right) \sim$ $\exp \left(\alpha^{2} t^{5} / 5\right)$ for large $t$, we need to begin and end the contour $C$ in sectors for which $\cos 5 \theta<0$ (setting $\left.t=|t| e^{i \theta}\right)$. There are five such sectors, specifically

$$
\begin{aligned}
& \theta \in \Theta_{n} \equiv\left[\frac{2 n \pi+\pi / 2}{5}, \frac{2 n \pi+3 \pi / 2}{5}\right] \\
& n=0,1,2,3,4,5 .
\end{aligned}
$$

Therefore, any contour which originates at one of them and terminates at another yields a solution to (12). One could then find four linearly independent functions of the form

$$
I_{i}(\rho)=\int_{C_{i}} \exp \left(\rho t+\frac{\alpha^{2} t^{5}}{5}-\frac{t^{3}}{3}\right) d t
$$

The asymptotic expression for $I_{i}(\rho)$ for large values of $\rho$ is obtained by evaluating the integral equation (26) by the method of steepest descents.

\section{Asymptotic Expansion}

First we briefly review the method of steepest descent to introduce some useful formulas. This technique is very powerful to calculate integrals of the form

$$
I(\rho)=\int_{C} g(z) e^{\rho f(z)} d z
$$

where $C$ is a contour in the complex plane and $g(z)$ and $f(z)$ are analytic functions. The parameter $\rho$ is real and we are usually interested in the behaviors of $I(\rho)$ as $\rho \rightarrow$ $\pm \infty$. The key step of the method of steepest descent is applying Cauchy's theorem to deform the contours $C$ to the contours consisting of steepest descent paths and other paths joining endpoints of two different steepest descent paths if necessary. Usually, the joining paths are chosen to make negligible contributions to $I(\rho)$. It is easy to show that $\operatorname{Im} f(z)$ is constant along steepest descent paths. When a steepest descent contour passes through a saddle point $z_{0}$, where $f^{\prime}\left(z_{0}\right)=0, f(z)$ and $g(z)$ are expanded around $z_{0}$ and Watson's lemma is used to determine asymptotic behaviors of $I(\rho)$. Specifically, consider a contour $C$ through a saddle point $z_{0}$. A new variable $\tau$ is introduced as $\tau=f(z)-f\left(z_{0}\right)$ to calculate $I(\rho)$. The saddle point $z_{0}$ divides the contour $C$ into two contours $C_{1}$ and $C_{2}$. Generally, $\tau$ monotonically increases from $-\infty$ to zero along one contour, say $C_{1}$, and monotonically decreases from zero to $-\infty$ along $C_{2}$. Thus, the integral becomes

$$
\begin{aligned}
& I(\rho)=\exp \left[\rho f\left(z_{0}\right)\right]\left[\left.\int_{-\infty}^{0} g(\tau) \exp [\rho \tau] \frac{d z}{d \tau}\right|_{C_{1}} d \tau\right. \\
& \left.+\left.\int_{0}^{-\infty} g(\tau) \exp [\rho \tau] \frac{d z}{d \tau}\right|_{C_{2}} d \tau\right] .
\end{aligned}
$$

The physically acceptable solution can be represented by an integral

$$
I(\rho)=\int_{C} \exp \left(\rho t+\frac{\alpha^{2} t^{5}}{5}-\frac{t^{3}}{3}\right) d t
$$

where $C$ is any contour which ranges from $t=\exp (-3 \pi i / 5) \infty$ to $t=\exp (3 \pi i / 5) \infty$. In fact, as we show later in the section for positive $\rho$, there exists a steepest descent contour from $t=\exp (-3 \pi i / 5) \infty$ to $t=\exp (3 \pi i / 5) \infty$, to which $C$ can be deformed. Moreover, the integral on such a steepest descent contour yields the required asymptotic behavior of $I(\rho)$ at large positive value of $\rho$. Here the exponent in the integrand has movable saddle points. Making the change of variables $t=|\rho|^{1 / 2} s$, one gets

$$
\begin{aligned}
& I(\rho)=|\rho|^{1 / 2} \\
& \cdot \int_{\exp (-3 \pi i / 5) \infty}^{\exp (3 \pi i / 5) \infty} \exp \left[|\rho|^{3 / 2}\left( \pm s+\frac{a s^{5}}{5}-\frac{s^{3}}{3}\right)\right] d s \\
& \quad \equiv|\rho|^{1 / 2} \int_{\exp (-3 \pi i / 5) \infty}^{\exp (3 \pi i / 5) \infty} \exp \left[|\rho|^{3 / 2} f_{ \pm}(s)\right] d s,
\end{aligned}
$$

where + for $\rho>0$ and - for $\rho<0$ and $a=\alpha^{2}|\rho| \ll 1$ in the physical region.

\subsection{Large Positive $\rho$. For $\rho>0$, we have}

$$
f_{+}(s)=s+\frac{a s^{5}}{5}-\frac{s^{3}}{3}
$$

There are four saddle points given by $f_{+}^{\prime}(s)=0$ at

$$
\begin{aligned}
& s= \pm \lambda_{+} \equiv \pm \frac{\sqrt{1-\sqrt{1-4 a}}}{\sqrt{2 a}} \\
& s= \pm \eta_{+} \equiv \pm \frac{\sqrt{1+\sqrt{1-4 a}}}{\sqrt{2 a}} .
\end{aligned}
$$

Our goal now is to find a steepest descent contour emerging from $s=\exp (-3 \pi i / 5) \infty$ to $s=\exp (3 \pi i / 5) \infty$. We will show that such a contour passes through $s=-\lambda_{+}$. To find the contour we substitute $s=u+i v$ and identify the real and imaginary parts of $f_{+}(s)$ :

$$
\begin{aligned}
f_{+}(s)= & u\left(1-\frac{u^{2}}{3}+\frac{a u^{4}}{5}+v^{2}-2 a u^{2} v^{2}+a v^{4}\right) \\
& +i v\left(1-u^{2}+a u^{4}+\frac{v^{2}}{3}-2 a u^{2} v^{2}+\frac{a v^{4}}{5}\right) .
\end{aligned}
$$

Since $\operatorname{Im} f_{+}\left(-\lambda_{+}\right)=0$, the constant-phase contours passing through $s=-\lambda_{+}$must satisfy

$$
v\left(1-u^{2}+a u^{4}+\frac{v^{2}}{3}-2 a u^{2} v^{2}+\frac{a v^{4}}{5}\right)=0 .
$$


Therefore, one of the constant-phase contours passing through $s=-\lambda_{+}$is

$$
\begin{aligned}
C & :-\frac{1}{\sqrt{2 a}} \sqrt{1+2 a v^{2}-\sqrt{1-4 a+\frac{8}{3} a v^{2}+\frac{16}{5} a^{2} v^{4}}} \\
& +i v, \quad \text { for }-\infty<v<\infty,
\end{aligned}
$$

which is a steepest descent contour. In fact, around the saddle point $s=-\lambda_{+}$, one finds on the contour $C$

$$
s \sim-\lambda_{+}+b v^{2}+i v
$$

and hence

$$
f_{+}(s)=f_{+}\left(-\lambda_{+}\right)-\frac{v^{2}}{2} f_{+}^{\prime \prime}\left(-\lambda_{+}\right)+\mathcal{O}\left(v^{3}\right),
$$

where $b$ is a positive real number. Since $f_{+}^{\prime \prime}\left(-\lambda_{+}\right)$is real and positive, the contour $C$ is indeed a steepest descent contour. Note that $C$ goes to $s=\exp (-3 \pi i / 5) \infty$ as $v \rightarrow-\infty$ and $s=\exp (3 \pi i / 5) \infty$ as $v \rightarrow \infty$. In order to evaluate asymptotic expansion of $I(\rho)$, we break up the contour $C$ into $C_{1}$ and $C_{2}$, where $C_{1}\left(C_{2}\right)$ is the contour above (below) of $s=-\lambda_{+}$. Define

$$
\tau=f_{+}(s)-f_{+}\left(-\lambda_{+}\right),
$$

where $\tau$ monotonically decreases from zero to $-\infty$ as one moves away from $s=-\lambda_{+}$along $C_{1}$ to $s=\exp (3 \pi i / 5) \infty$ and along $C_{2}$ to $s=\exp (-3 \pi i / 5) \infty$, respectively. Since $f_{+}^{\prime}\left(-\lambda_{+}\right)=$ 0 , the expression for $s$ in terms of $\tau$ can be expressed as a power series of $\sqrt{-\tau}$. Then, noting that $-\tau=( \pm \sqrt{-\tau})^{2}$, one has

$$
s=-\lambda_{+}+\sum_{j=1}^{\infty} a_{j}( \pm \sqrt{-\tau})^{j},
$$

where $a_{i}$ can be obtained by substituting (39) into (38) and equating powers of $\sqrt{-\tau}$ on both sides of the equations. It is easy to find

$$
a_{1}=i \sqrt{\frac{2}{\left|f_{+}^{\prime \prime}\left(-\lambda_{+}\right)\right|}},
$$

where one finds $\operatorname{Im} a_{1}>0$. The contour $C_{1}$ is in the second quadrant and hence + sign is chosen in (39) for $C_{1}$. Therefore,

$$
\begin{aligned}
& \rho^{1 / 2} \int_{C_{1}} \exp \left[\rho^{3 / 2} f_{+}(s)\right] d s \\
& \quad=\rho^{1 / 2} \exp \left[\rho^{3 / 2} f_{+}\left(-\lambda_{+}\right)\right] \int_{0}^{-\infty} \exp \left(\rho^{3 / 2} \tau\right) \frac{d s}{d \tau} d \tau \\
& \quad \sim \exp \left[\rho^{3 / 2} f_{+}\left(-\lambda_{+}\right)\right] \sum_{j=1}^{\infty} \frac{j a_{j}}{2 \rho^{(3 j-2) / 4}} \Gamma\left(\frac{j}{2}\right) .
\end{aligned}
$$

For the contour segment $C_{2}$, the sign of $\sqrt{\tau}$ occurring in (39) has to be reversed. Moreover, the limit of integration on $C_{2}$ in the variable $\tau$ ranges from $-\infty$ to 0 . Thus,

$$
\begin{aligned}
& \rho^{1 / 2} \int_{C_{1}} \exp \left[\rho^{3 / 2} f_{+}(s)\right] d s \\
& \quad \sim-\exp \left[\rho^{3 / 2} f_{+}\left(-\lambda_{+}\right)\right] \sum_{j=1}^{\infty} \frac{(-1)^{j} j a_{j}}{2 \rho^{(3 j-2) / 4}} \Gamma\left(\frac{j}{2}\right) .
\end{aligned}
$$

Combining (41) and (42), we easily find

$$
\begin{aligned}
& I\left(1 \ll \rho \ll \alpha^{-2}\right) \\
& \quad \sim \frac{\exp \left[\rho^{3 / 2} f_{+}\left(-\lambda_{+}\right)\right]}{\rho^{1 / 4}} \sum_{j=0}^{\infty} \frac{(2 j+1) a_{2 j+1}}{\rho^{3 j / 2}} \Gamma\left(j+\frac{1}{2}\right) .
\end{aligned}
$$

3.2. Large Negative $\rho$. As for $\rho<0$, the exponent in the integrand of $I(\rho)$ is

$$
f_{-}(s)=-s+\frac{a s^{5}}{5}-\frac{s^{3}}{3} \text {. }
$$

Thus, one as well finds four saddle points given by $f_{+}^{\prime}(s)=0$ :

$$
\begin{aligned}
& s= \pm \lambda_{-} \equiv \pm \frac{\sqrt{1-\sqrt{1+4 a}}}{\sqrt{2 a}} \\
& s= \pm \eta_{-} \equiv \pm \frac{\sqrt{1+\sqrt{1+4 a}}}{\sqrt{2 a}}
\end{aligned}
$$

As before, our objective is to find steepest descent contours passing through the saddle point(s) in (45) that emerges from $s=\exp (-3 \pi i / 5) \infty$ to $s=\exp (3 \pi i / 5) \infty$. Substituting $s=$ $u+i v$, we obtain the real and imaginary parts of $f_{-}(s)$ :

$$
\begin{aligned}
& f_{-}(s) \\
& =u\left(-1-\frac{u^{2}}{3}+\frac{a u^{4}}{5}+v^{2}-2 a u^{2} v^{2}+a v^{4}\right) \\
& \quad+i v\left(-1-u^{2}+a u^{4}+\frac{v^{2}}{3}-2 a u^{2} v^{2}+\frac{a v^{4}}{5}\right) .
\end{aligned}
$$

We have already shown that only one steepest descent contour passing through $s=-\lambda_{+}$is sufficient to evaluate asymptotic behavior of $I(\rho)$ for large and positive $\rho$. However, for large and negative $\rho$, things are a little bit more complicated. Instead of one steepest descent contour, it turns out that we need three steepest descent contours passing through $\pm \lambda_{-}$and $\eta_{-}$, respectively, to connect two endpoints at $s=\exp ( \pm 3 \pi i / 5) \infty$.

First consider the steepest descent contour through $s=$ $-\lambda_{-}$. Since $f_{+}\left(-\lambda_{-}\right)$is a pure imaginary number, the steepest descent contour must satisfy

$$
\begin{aligned}
& i v\left(-1-u^{2}+a u^{4}+\frac{v^{2}}{3}-2 a u^{2} v^{2}+\frac{a v^{4}}{5}\right) \\
& \quad=f_{-}\left(-\lambda_{-}\right) .
\end{aligned}
$$

Solutions to the last equation give us a constant phase contour $C_{-\lambda_{-}}$passing through $s=-\lambda_{-}$, which emanates from 
$s=\exp (-3 \pi i / 5) \infty$ and finally approaches $s=\exp (-\pi i / 5) \infty$. The contour $C_{-\lambda_{-}}$actually is composed of three segments as

$$
\begin{aligned}
& C_{-\lambda_{-}, 1}:-\frac{1}{\sqrt{2 a}} \sqrt{1+2 a v^{2}-\sqrt{F_{-\lambda_{-}}(v)}}+i v, \\
& \text { for }-\infty<v<-\operatorname{Im} \lambda_{-}, \\
& C_{-\lambda_{-}, 2}: \frac{1}{\sqrt{2 a}} \sqrt{1+2 a v^{2}-\sqrt{F_{-\lambda_{-}}(v)}}+i v, \\
& \text { for }-\operatorname{Im} \lambda_{-}<v<-v_{0}, \\
& C_{-\lambda_{-}, 3}: \frac{1}{\sqrt{2 a}} \sqrt{1+2 a v^{2}+\sqrt{F_{-\lambda_{-}}(v)}}+i v, \\
& \text { for }-v_{0}>v>-\infty,
\end{aligned}
$$

where we define

$$
F_{ \pm \lambda_{-}}(v)=1+4 a+\frac{8}{3} a v^{2}+\frac{16}{5} a^{2} v^{4}+\frac{4 a f_{-}\left( \pm \lambda_{-}\right)}{i v},
$$

and $v_{0}$ is a solution to $F_{+\lambda_{-}}(v)=0$ that satisfies $0<v_{0} \ll$ 1. It is straightforward to verify that, along $C_{-\lambda}, \operatorname{Re} f_{-}(s)$ monotonically increases from $-\infty$ to 0 as one moves from $s=\exp (-3 \pi i / 5) \infty$ to $s=-\lambda_{-}$and then monotonically decreases from 0 to $-\infty$ as one moves away from $s=-\lambda_{-}$ to $s=\exp (-\pi i / 5) \infty$. Hence, the contour $C_{-\lambda}$ is indeed the steepest descent contour passing through $s=-\lambda_{-}$. Now we calculate the contour integral on $C_{-\lambda_{-}}$. Introduce

$$
\tau=f_{-}(s)-f_{-}\left(-\lambda_{-}\right),
$$

where $\tau$ is real on $C_{-\lambda_{-}}$and varies from $-\infty$ to zero and then to $-\infty$ along $C_{-\lambda_{-}}$. Then, one has

$$
s=-\lambda_{-}+\sum_{j=1}^{\infty} b_{j}( \pm \sqrt{-\tau})^{j},
$$

where $b_{i}$ can be obtained by substituting (50) into (51). One easily gets

$$
b_{1}=\exp \left(\frac{\pi}{4} i\right) \sqrt{\frac{2}{\left|f_{-}^{\prime \prime}\left(-\lambda_{+}\right)\right|}} .
$$

Since Re $\exp ((\pi / 4) i)>0$, one has $-\sqrt{-\tau}$ for $C_{-\lambda_{-}, 1}$ and $\sqrt{-\tau}$ for $C_{-\lambda_{-}, 2}+C_{-\lambda_{-}, 3}$ in (51). Therefore,

$$
\begin{aligned}
& |\rho|^{1 / 2} \int_{C_{-\lambda_{-}}} \exp \left[|\rho|^{3 / 2} f_{-}(s)\right] d s \sim 2 \rho^{1 / 2} \\
& \cdot \exp \left[|\rho|^{3 / 2} f_{-}\left(-\lambda_{-}\right)\right] \\
& \cdot \int_{0}^{-\infty} \exp \left(|\rho|^{3 / 2} \tau\right) \sum_{j=0}^{\infty}(2 j+1) b_{j}(\sqrt{-\tau})^{2 j} d \sqrt{-\tau} \\
& =\frac{\exp \left[|\rho|^{3 / 2} f_{-}\left(-\lambda_{-}\right)\right]}{|\rho|^{1 / 4}} \sum_{j=0}^{\infty} \frac{(2 j+1) b_{j}}{|\rho|^{3 j / 2}} \Gamma\left(j+\frac{1}{2}\right) .
\end{aligned}
$$

Analogously, one can readily write down a constant phase contour $C_{+\lambda_{-}}$passing through $s=-\lambda_{-}$, which starts from $s=\exp (\pi i / 5) \infty$ and ends at $s=\exp (3 \pi i / 5) \infty$. As before, $C_{+\lambda_{-}}$consists of three segments:

$$
\begin{gathered}
C_{+\lambda_{-}, 1}: \frac{1}{\sqrt{2 a}} \sqrt{1+2 a v^{2}+\sqrt{F_{+\lambda_{-}}(v)}}+i v, \\
\text { for }+\infty>v>v_{0}, \\
C_{+\lambda_{-}, 2}: \frac{1}{\sqrt{2 a}} \sqrt{1+2 a v^{2}-\sqrt{F_{+\lambda_{-}}(v)}}+i v, \\
\text { for } v_{0}>v>\operatorname{Im} \lambda_{-}, \\
C_{+\lambda_{-}, 3}:-\frac{1}{\sqrt{2 a}} \sqrt{1+2 a v^{2}-\sqrt{F_{+\lambda_{-}}(v)}}+i v,
\end{gathered}
$$$$
\text { for } \operatorname{Im} \lambda_{-}<v<+\infty \text {. }
$$

It is also straightforward to verify that $C_{+\lambda_{-}}$is a steepest descent contour as well. Setting

$$
\tau=f_{-}(s)-f_{-}\left(\lambda_{-}\right),
$$

one finds that $\tau$ is real on $C_{+\lambda}$ and varies from $-\infty$ to zero and then to $-\infty$ along $C_{+\lambda_{-}}$. Note that $f_{+}(s)$ is an odd function and $\lambda_{-}^{*}=-\lambda_{-}$. Taking complex conjugate of both sides of (55), one then has on $C_{+\lambda_{-}}$

$$
s=\lambda_{-}+\sum_{j=1}^{\infty} b_{j}^{*}( \pm \sqrt{-\tau})^{j} .
$$

Since $\operatorname{Re} b_{1}^{*}>0$, one has $\sqrt{-\tau}$ for $C_{+\lambda_{-}, 1}+C_{+\lambda_{-, 2}}$ and $-\sqrt{-\tau}$ for $C_{+\lambda_{-}, 3}$ in (56). Therefore,

$$
\begin{aligned}
& |\rho|^{1 / 2} \int_{C_{+\lambda_{-}}} \exp \left[|\rho|^{3 / 2} f_{-}(s)\right] d s \\
& \sim-\frac{\exp \left[|\rho|^{3 / 2} f_{-}\left(\lambda_{-}\right)\right]}{|\rho|^{1 / 4}} \sum_{j=0}^{\infty} \frac{b_{j}^{*}(2 j+1)}{|\rho|^{3 j / 2}} \Gamma\left(j+\frac{1}{2}\right) .
\end{aligned}
$$

Since the values of $\operatorname{Im} f_{-}(s)$ are different on $C_{ \pm \lambda_{-}}$, it is obvious that we need a third contour which joins $C_{ \pm \lambda}$ up at $s=\exp ( \pm \pi i / 5) \infty$, respectively. Here, we consider a constant phase contour $C_{\eta_{-}}$connecting $s=\exp (-\pi i / 5) \infty$ to $\exp (\pi i / 5) \infty$ that passes through $\eta_{-}$. Since $\operatorname{Im} f(s)=$ $\operatorname{Im} f_{-}\left(\eta_{-}\right)=0$ on the contour $C_{\eta_{-}}$, one finds that

$$
\begin{aligned}
& C_{\eta_{-}}: \frac{1}{\sqrt{2 a}} \sqrt{1+2 a v^{2}+\sqrt{1+4 a+\frac{8}{3} a v^{2}+\frac{16}{5} a^{2} v^{4}}} \\
& \quad+i v, \quad \text { for }-\infty<v<+\infty,
\end{aligned}
$$

is a curve of steepest descent. On $C_{\eta_{-}}$, define

$$
\tau=f_{-}(s)-f_{-}\left(\eta_{-}\right),
$$


which is real on $C_{\eta_{-}}$and varies from $-\infty$ to zero and then to $-\infty$ along $C_{\eta_{-}}$. Then, one finds

$$
\begin{aligned}
& s=\lambda_{-}+\sum_{j=1}^{\infty} c_{j}( \pm \sqrt{-\tau})^{j}, \\
& c_{1}=i \sqrt{\frac{2}{\left|f_{-}^{\prime \prime}\left(\eta_{-}\right)\right|}} .
\end{aligned}
$$

Similarly, we break up the contour $C_{\eta_{-}}$into $C_{\eta_{-}, 1}$ and $C_{\eta_{-}, 2}$, where $C_{\eta_{-}, 1}\left(C_{\eta_{-}, 2}\right)$ is the contour above (below) of $s=\eta_{-}$ with $\sqrt{-\tau}$ for $C_{\eta_{-}, 1}$ and $-\sqrt{-\tau}$ for $C_{\eta_{-}, 2}$ in (60). Thus,

$$
\begin{aligned}
& |\rho|^{1 / 2} \int_{C_{\eta_{-}}} \exp \left[|\rho|^{3 / 2} f_{-}(s)\right] d s \\
& \sim \frac{\exp \left[|\rho|^{3 / 2} f_{-}\left(\eta_{-}\right)\right]}{|\rho|^{1 / 4}} \sum_{j=0}^{\infty} \frac{(2 j+1) c_{2 j+1}}{|\rho|^{3 j / 2}} \Gamma\left(j+\frac{1}{2}\right) .
\end{aligned}
$$

Note that although paths $C_{\eta_{-}}$and $C_{ \pm \lambda_{-}}$never join up at $s=$ $\exp ( \pm \pi i / 5) \infty$, the integrand $\exp \left[f_{-}(s)\right] \sim \exp \left(\left(a|\rho|^{3 / 2} / 5\right) s^{5}\right)$ tends to zero exponentially. Therefore, there is no contribution from a connecting path from $C_{\eta_{-}}$and $C_{ \pm} \lambda_{-}$at a distance $R$ from the origin in the limit $R \rightarrow \infty$. As a result, the integral $I(\rho)$ equals the sum of three contour integrals on the different steepest descent curves $C_{\eta_{-}}$and $C_{ \pm \lambda_{-}}$. Combining (53), (57), and (62) gives the full asymptotic expansion of $I(\rho)$ for large and negative $\rho$ :

$$
\begin{aligned}
& I\left(-1 \gg \rho \gg-\alpha^{-2}\right) \\
& \sim 2 i \sum_{j=0}^{\infty} \frac{\operatorname{Im}\left(\exp \left[-|\rho|^{3 / 2} f_{-}\left(\lambda_{-}\right)\right] b_{j}\right)}{|\rho|^{1 / 4}} \frac{(2 j+1)}{|\rho|^{3 j / 2}} \Gamma(j \\
& \left.+\frac{1}{2}\right)+\frac{\exp \left[|\rho|^{3 / 2} f_{-}\left(\eta_{-}\right)\right]}{|\rho|^{1 / 4}} \sum_{j=0}^{\infty} \frac{(2 j+1) c_{2 j+1}}{|\rho|^{3 j / 2}} \Gamma(j \\
& \left.+\frac{1}{2}\right) .
\end{aligned}
$$

\section{WKB Approximation}

The authors of [22] find the WKB approximation in deformed space with minimal length. In [22], they consider the deformed commutation relation

$$
[X, P]=i \hbar f(P),
$$

where $f(P)$ is an arbitrary function of $P$. In our paper, we set $f(P)=1+\beta P^{2}$. Defining $P(p)$,

$$
\frac{d P(p)}{d p}=f(P),
$$

and $p(P)$ an inverse function of $P(p)$, they find the physical optics approximation to the solution of the deformed Schrodinger equation,

$$
P^{2}\left(\frac{\hbar}{i} \frac{d}{d x}\right) \psi(x)+2 m[V(x)-E] \psi(x)=0,
$$

is

$$
\begin{aligned}
& \psi(x)=\frac{1}{\sqrt{|P(x) f(P(x))|}}\left(C_{1} \exp \left[\frac{i}{\hbar} \int^{x} p(x) d x\right]\right. \\
& \left.+C_{2} \exp \left[-\frac{i}{\hbar} \int^{x} p(x) d x\right]\right),
\end{aligned}
$$

where $P(x)=\sqrt{2 m(E-V(x))}$ and $p(x)=p(P(x))$ in (67). It is also shown there that if (67) is valid, the condition

$$
\left|P^{2}(x)\right| \gg \hbar\left|\frac{d}{d x} P(x) f(P(x))\right|
$$

has to be satisfied. However, the condition equation (68) fails near a turning point where $P(x)=0$. Thus, if we want to determine bound state energies, we need to be able to match wave functions at the turning points. Here we consider a potential $V(x)$ with its classical turning point located at $x=0$. A linear approximation to the potential $V(x)$ near the turning point $x=0$ is

$$
V(x) \approx V(0)+F x
$$

where $F=V^{\prime}(0)$. The linearized potential (69) is discussed in the previous two sections. Our discussion shows that the parameter $\alpha=\ell_{\beta}\left(2 m|F| / \hbar^{2}\right)^{1 / 3}$ plays an important role in analyzing asymptotic behaviors of the solutions. When $\alpha \ll 1$, the physically acceptable solution can exist at large argument $\rho$, while condition (14) still holds. Accordingly, a turning points is called a smooth one if $\alpha=$ $\ell_{\beta}\left(2 m|F| / \hbar^{2}\right)^{1 / 3} \ll 1$. Otherwise, it is called a sharp turning point.

4.1. WKB Connection through a Smooth Turning Point. Now we want to match $\mathrm{WKB}$ wave functions at a smooth turning point in the deformed space with $f(P)=1+\beta P^{2}$ up to $\mathcal{O}(\beta)$. Suppose $x=0$ is a smooth turning point, which means $V(0)=E$, and $V>E$ for all $x>0$. The region to the left of the turning point is classically forbidden where the wave function must be damped and becomes zero at infinity. Thus, far from $x=0$, the wave function has the form

$$
\begin{aligned}
& \psi(x) \\
& =\frac{1}{\sqrt{|P(x) f(P(x))|}} C \exp \left[-\frac{1}{\hbar}\left|\int_{0}^{x} p(x) d x\right|\right]
\end{aligned}
$$

for $x>0$.

To the right of the turning point, the wave function is given by

$$
\begin{gathered}
\psi(x)=\frac{1}{\sqrt{|P(x) f(P(x))|}}\left(C_{1} \exp \left[\frac{i}{\hbar} \int_{0}^{x} p(x) d x\right]\right. \\
\left.+C_{2} \exp \left[-\frac{i}{\hbar} \int_{0}^{x} p(x) d x\right]\right), \quad \text { for } x<0 .
\end{gathered}
$$


Around the turning point, $x$ is small and $P(x) \sim \sqrt{2 m F} \sqrt{-x}$. In this region, we may approximate (70) and (71) by

$$
\begin{gathered}
\psi(x) \approx(2 m F \hbar)^{-1 / 3} x^{-1 / 4}\left(1+\frac{3 a}{4}+\mathcal{O}\left(a^{2}\right)\right) C \\
\cdot \exp \left[-\frac{2}{3 \hbar} x^{3 / 2}\left(1+\frac{3}{10} a+\mathcal{O}\left(a^{2}\right)\right)\right],
\end{gathered}
$$

for $x>0$,

$$
\begin{gathered}
\psi(x)=(2 m F \hbar)^{-1 / 3} x^{-1 / 4}\left(1-\frac{3 a}{4}+\mathcal{O}\left(a^{2}\right)\right) \\
\cdot\left(C_{1} \exp \left[\frac{2 i}{3 \hbar}|x|^{3 / 2}\left(1-\frac{3}{10} a+\mathcal{O}\left(a^{2}\right)\right)\right]\right. \\
\left.+C_{2} \exp \left[-\frac{2 i}{3 \hbar}|x|^{3 / 2}\left(1-\frac{3}{10} a+\mathcal{O}\left(a^{2}\right)\right)\right]\right),
\end{gathered}
$$

for $x<0$.

The criteria (68) for validity of the WKB approximation are satisfied if

$$
|x| \gg\left(\frac{2 m F}{\hbar^{2}}\right)^{-1 / 3}
$$

where we neglect $\beta P^{2}$ in derivation. On the other hand, when the potential is linearized around the turning point $x=0$, the Schrodinger equation becomes

$$
\frac{d^{2} \psi(x)}{d x^{2}}-\ell_{\beta}^{2} \frac{d^{4} \psi(x)}{d x^{4}}-\frac{2 m \mu x}{\hbar^{2}} \psi(x) \approx 0,
$$

where $\beta=3 \ell_{\beta}^{2} / 2 \hbar^{2}$. To solve the approximate differential equation, we make the substitution

$$
\rho=x\left(\frac{2 m F}{\hbar^{2}}\right)^{1 / 3}
$$

In terms of $\rho$, the solution to (75) which matches (72) and (73) in two different limits is actually $I(\rho)$ calculated in Section 3. Specifically, the solution is

$$
\psi(x)=D I(\rho)=D I\left(x\left(\frac{2 m F}{\hbar^{2}}\right)^{1 / 3}\right)
$$

where $D$ is a constant to be determined by asymptotic matching. It is easily shown from (74) that there exist overlap regions where both $\mathrm{WKB}$ approximation and (75) hold. In the overlap regions, one finds $|\rho| \gg 1$ and $|x| \ll 1$. Therefore, we approximate $I(\rho)$ by its leading asymptotic behaviors for large argument in the overlap regions. The appropriate formulas are

$$
\begin{aligned}
& I\left(1 \ll \rho \ll \alpha^{-2}\right) \sim \frac{i \sqrt{\pi}\left(1+3 a / 4+\mathcal{O}\left(a^{2}\right)\right)}{\rho^{1 / 4}} \\
& \cdot \exp \left[-\frac{2 \rho^{3 / 2}}{3}\left(1+\frac{3 a}{10}+\mathcal{O}\left(a^{2}\right)\right)\right], \\
& I\left(-1 \gg \rho \gg-\alpha^{-2}\right) \sim \frac{2 i \sqrt{\pi}\left(1-3 a / 4+\mathcal{O}\left(a^{2}\right)\right)}{|\rho|^{1 / 4}} \\
& \cdot \sin \left[\frac{2|\rho|^{3 / 2}}{3}\left(1-\frac{3 a}{10}+\mathcal{O}\left(a^{2}\right)\right)+\frac{\pi}{4}\right],
\end{aligned}
$$

where $\alpha \ll 1$ for a smooth turning point and $a=$ $\ell_{\beta}^{2}\left(2 m F / \hbar^{2}\right)^{2 / 3}|\rho| \ll 1$ as required by condition (13). Requiring that (78) and (79) match (72) and (73) in the overlap region, respectively, gives $C_{1}=-i C e^{i \pi / 4}$ and $C_{2}=$ $i C e^{i \pi / 4}$ up to $\mathcal{O}(\beta)$. In summary, in the overlap region, we find WKB solutions and the asymptotic values of the solution to the Schrodinger equation with a linear approximation to the potential $V(x)$. Then, by making (78) and (79) match (72) and (73), respectively, the $\mathrm{WKB}$ connection formula with the deformed commutator $[X, P]=i \hbar\left(1+\beta P^{2}\right)$ is obtained up to $\mathcal{O}(\beta)$. The connection formula around a smooth turning point is put in a way that

$$
\begin{aligned}
& \frac{C}{\sqrt{|P(x) f(P(x))|}} \exp \left(-\frac{1}{\hbar}\left|\int_{0}^{x} p(x) d x\right|\right) \\
& \longrightarrow \frac{2 C}{\sqrt{|P(x) f(P(x))|}} \sin \left(\frac{1}{\hbar} \int_{0}^{x} p(x) d x+\frac{\pi}{4}\right),
\end{aligned}
$$

up to $\mathcal{O}\left(\beta^{2}\right)$,

which is directional, just as in ordinary quantum mechanics [25]. The analysis always proceeds from classically forbidden region to classically allowed one. For bound states, the uniqueness of the wave function in the classically allowed region leads to the Bohr-Sommerfeld quantization condition

$$
\int_{a}^{b} p(x) d x=\left(n+\frac{1}{2}\right) \pi \hbar, \quad \text { up to } \mathcal{O}\left(\beta^{2}\right),
$$

where $a$ and $b$ are two smooth turning points for the potential $V(x)$. Notice that although (81) is claimed in [22], one still needs to obtain the connection formula to derive (81) rigorously, which is not presented in [22].

\subsection{Discussion}

4.2.1. Sharp Turning Point. Near a sharp turning point $x=0$, not only the $\mathrm{WKB}$ approximation is no longer valid but also matching the two $\mathrm{WKB}$ solutions across the turning point stops making sense. In fact, from the previous subsection, one finds that the asymptotic matching is valid as long as there 
exists an overlap region where $1 \ll|\rho| \ll \alpha^{-2}$. However, such region does not exist unless $\alpha \ll 1$, which means that the asymptotic matching fails through a sharp turning point.

It can be shown, through (68), that WKB approximations are valid as long as $|x| \gg\left(2 m|F| / \hbar^{2}\right)^{-1 / 3}$ in the region where the potential is approximated by a linear one. To put it another way, if there exists a region where both $\mathrm{WKB}$ and linear approximations are valid, one finds $|x| \gg\left(2 m|F| / \hbar^{2}\right)^{-1 / 3}$ for such a region. When $|x| \gg\left(2 m|F| / \hbar^{2}\right)^{-1 / 3}$, we have

$$
\left|\beta P^{2}\right| \approx \frac{\ell_{\beta}^{2}}{\left(2 m|F| / \hbar^{2}\right)^{2 / 3}} \frac{x}{\left(2 m|F| / \hbar^{2}\right)^{1 / 3}} \gg 1
$$

for a sharp turning point. However, $\left|\beta P^{2}\right| \ll 1$ is required by the GUP model. This means that, as moving away from the sharp turning point, one is far beyond the region where the linear approximation to the potential is good before reaching the WKB valid region. One might resort to a higher order approximation to the potential and asymptotic matching in the overlap region to find WKB connection formula through a sharp turning point.

4.2.2. $\mathcal{O}(\beta)$ versus $\mathcal{O}(\hbar)$. When $\hbar$ can be regarded as a small quantity, the approximate solution to the deformed Schrodinger equation

$$
\frac{d^{2} \psi(x)}{d x^{2}}-\frac{2 \hbar^{2} \beta}{3} \frac{d^{4} \psi(x)}{d x^{4}}+\frac{2 m(E-V(x))}{\hbar^{2}} \psi(x)=0
$$

is easy to find using WKB analysis. To be specific, the approximate solution is expressed in an exponential power series of the form

$$
\psi(x) \sim \exp \left[\frac{1}{\hbar} \sum_{n=0}^{\infty} \hbar^{n} S_{n}(x)\right] .
$$

The authors of [22] find

$$
S_{1}=-\frac{1}{2} \ln |2 P f(P)| .
$$

Since here $f(P)=1+\beta P^{2}$, we have for $S_{1}$

$$
S_{1} \approx \ln \frac{1}{\sqrt{|P|}}-\frac{\beta}{2} P^{2}+\mathcal{O}\left(\beta^{2}\right) .
$$

Moreover, the leading order (in terms of $\beta$ ) of $S_{2}$ is just WKB $\mathcal{O}\left(\hbar^{2}\right)$ correction calculated in the ordinary quantum mechanics. Therefore, we obtain [27]

$$
S_{2} \approx \frac{P^{\prime}}{4 P^{2}}+\int \frac{P^{\prime 2}}{8 P^{3}} d x+\mathcal{O}(\beta) .
$$

If one uses WKB approximations to evaluate quantum gravity induced corrections, say to energy levels or tunnelling rates, one may want to have

$$
\beta P^{2} \gtrsim \hbar S_{2}
$$

Otherwise, the quantum gravity correction $(\sim \mathcal{O}(\beta))$ on the first-order WKB approximation $\left(\sim \mathcal{O}\left(\hbar^{0}\right)\right)$ could be overwhelmed by the second-order WKB approximation $(\sim \mathcal{O}(\hbar))$. Suppose $a$ is the characteristic length of the potential $V(x)$, for example, the width of a square-well potential. Then we can get a rough estimate on $S_{2}$ :

$$
\hbar S_{2} \sim \frac{\hbar}{a P} \sim \frac{\lambda}{a},
$$

where $\lambda$ is the de Broglie wavelength of a particle with momentum $P$. As a result, condition (88) becomes

$$
\begin{aligned}
\frac{\ell_{\beta}^{2}}{\lambda^{2}} & \gtrless \frac{\lambda}{a} \Longrightarrow \\
\lambda & \leqslant \ell_{\beta}\left(\frac{a}{\ell_{\beta}}\right)^{1 / 3} .
\end{aligned}
$$

It is interesting to note that condition (90) is a rough estimate and a more accurate estimate could be obtained once the form of the potential is given.

Taking into account the constraints (90) on the de Broglie wavelength $\lambda$ of a particle, one may conclude that the WKB approximation is not a powerful tool to calculate quantum gravity corrections unless the energy of the particle considered is high enough. However, there is an exception if the corresponding Schrodinger equation in the ordinary quantum mechanics can be solved exactly. In this case, $\mathcal{O}(\beta)$ corrections calculated on the WKB first-order approximation are just quantum gravity corrections to exact results up to $\mathcal{O}(\beta) \mathcal{O}\left(\hbar^{0}\right)$ even without having $(90)$ required. For example, if we employ WKB analysis to calculate the energy spectrum of a bound state in the deformed space, the energy levels can be represented by a series in powers of $\hbar$ :

$$
E_{n}=\sum_{j=0}^{\infty} \hbar^{j} E_{n, j}(\beta)
$$

where $E_{n, j}(\beta)$ can be expanded in terms of $\beta$ :

$$
E_{n, j}(\beta)=\sum_{k=0}^{\infty} \beta^{k} E_{n, j}^{k} .
$$

If on the first-order WKB approximation one calculates $E_{n, 0}(\beta)$ up to $\mathcal{O}(\beta)$,

$$
E_{n, 0}(\beta)=E_{n, 0}^{0}+\beta E_{n, 0}^{1}+\mathcal{O}\left(\beta^{2}\right),
$$

the energy levels are

$$
E_{n}=E_{n, 0}^{0}+\beta E_{n, 0}^{1}+\mathcal{O}\left(\beta^{2}\right)+\mathcal{O}(\hbar) .
$$

In order to have (94) making sense, one requires $\beta E_{n, 0}^{1} \gtrsim$ $\mathcal{O}(\hbar)$. On the other hand, if we know the exact result $E_{n}$ with $\beta=0$, namely, $E_{n}^{(0)}$,

$$
E_{n}^{(0)}=\sum_{j=0}^{\infty} \hbar^{j} E_{n, j}^{0}
$$


equation (91) becomes

$$
E_{n}=E_{n}^{(0)}+\beta E_{n, 0}^{1}+\mathcal{O}(\hbar) \mathcal{O}(\beta)+\mathcal{O}\left(\beta^{2}\right)
$$

Since $\mathcal{O}(\hbar) \mathcal{O}(\beta)$ is automatically smaller than $\beta E_{0}^{1},(96)$ always makes sense as long as $\mathcal{O}(\hbar) \ll 1$.

To illustrate our points, we use the WKB approximation to derive the energy levels of a particle confined to the onedimensional potential $V(x)=F|x|$ whose turning points are

$$
\begin{aligned}
& a=-\frac{E}{F}, \\
& b=\frac{E}{F} .
\end{aligned}
$$

The energy quantization condition (81) from first-order WKB approximation then becomes

$$
\begin{aligned}
\ell_{F}^{-3 / 2} & \int_{-E / F}^{E / F} \sqrt{\frac{E}{F}-|x|} d x \\
& -\frac{\ell_{\beta}^{2}}{2 \ell_{F}^{9 / 2}} \int_{-E / F}^{E / F}\left(\frac{E}{F}-|x|\right)^{3 / 2} d x \\
= & \left(n+\frac{1}{2}\right) \pi+\mathcal{O}\left(\beta^{2}\right),
\end{aligned}
$$

where $\ell_{F}=\left(\hbar^{2} / 2 m F\right)^{1 / 3}$ is the characteristic length of the potential $V(x)=F|x|$. From the last equation, we obtain

$$
\frac{E_{n}}{F} \approx \ell_{n}\left(1+\frac{\ell_{\beta}^{2} \ell_{n}}{5 \ell_{F}^{3}}+\mathcal{O}\left(\beta^{2}\right)+\mathcal{O}(\hbar)\right),
$$

where $\ell_{n}=\ell_{F}[(3 / 4)(n+1 / 2) \pi]^{2 / 3}$. What is $\mathcal{O}(\hbar)$ ? The secondorder generalization of (81) with $\beta=0$ is given in [25]:

$$
\begin{aligned}
& \ell_{F}^{-3 / 2} \int_{-E^{(0)} / F}^{E^{(0)} / F} \sqrt{\frac{E^{(0)}}{F}-|x|} d x+\frac{F_{F}^{3 / 2} \ell^{3 / 2}}{48 E^{(0) 3 / 2}} \\
& =\left(n+\frac{1}{2}\right) \pi+\mathcal{O}\left(\hbar^{2}\right),
\end{aligned}
$$

which gives

$$
\frac{E_{n}^{(0)}}{F} \approx \ell_{n}\left(1-\frac{\ell_{F}^{3}}{96 \ell_{n}^{3}}+\mathcal{O}\left(\hbar^{2}\right)\right) .
$$

We can then estimate $\mathcal{O}(\hbar)$ through (101):

$$
\mathcal{O}(\hbar) \sim \frac{\ell_{F}^{3}}{\ell_{n}^{3}},
$$

which can also be easily obtained by dimensional analysis. If one wants the first-order approximation (99) to make sense, the second term in (99) should be comparable to or larger than $\mathcal{O}(\hbar)$ and then one gets

$$
\ell_{n} \geq \ell_{F} \sqrt{\frac{\ell_{F}}{\ell_{\beta}}} .
$$

The de Broglie wavelength of a particle with energy $E_{n} \sim F \ell_{n}$ is

$$
\lambda_{n} \sim \frac{\hbar}{\sqrt{2 m F \ell_{n}}} \sim \frac{\ell_{F}^{3 / 2}}{\sqrt{\ell_{n}}} .
$$

Thus, inequality (103) reads

$$
\lambda_{n} \leqslant \ell_{F}\left(\frac{\ell_{\beta}}{\ell_{F}}\right)^{1 / 4}
$$

which is much milder than (90). In a practical way, $\hbar$ and $\beta$ can be expressed in terms of $\ell_{\beta}, \ell_{F}$ and $\ell_{n}$. In fact, it is easily shown that

$$
\begin{aligned}
& \mathcal{O}\left(\hbar^{m}\right) \sim \mathcal{O}\left(\frac{\ell_{F}^{3 m}}{\ell_{n}^{3 m}}\right) \sim \mathcal{O}\left(\frac{1}{n^{2 m}}\right), \\
& \mathcal{O}\left(\beta^{m}\right) \sim \mathcal{O}\left(\frac{\ell_{\beta}^{2 m}}{\ell_{F}^{2 m}}\right) .
\end{aligned}
$$

4.3. Application. The dimensionless number $\beta_{0}=c^{2} m_{p l}^{2} \beta=$ $\hbar^{2} \beta / \ell_{p}^{2}$ plays an important role when implications and applications of nonzero minimal length are discussed. Normally, if the minimal length is assumed to be order of the Planck length $\ell_{p}$, one has $\beta_{0} \sim 1$. In [12], based on the precision measurement of Lamb shift, an upper bound of $\beta_{0}$ was given by $\beta_{0}<10^{36}$. The authors in [28] placed constraints on $\beta_{0}$ from the precession of the perihelion of the Mercury, which was $\beta_{0}<10^{-66}$. However, as pointed out in [29], the effective deformation parameter was substantially reduced by a factor $N^{-2}$ for a macroscopic body which consists of $N$ quarks. Thus, an upper bound on $\beta_{0}$ for quarks was $\beta_{0}^{q}<10^{36}$. In the following, we first use the Hamilton-Jacobi method to study the effects of the minimal length on the classical motions. The Bohr-Sommerfeld quantization is then used to investigate statistical physics in deformed spaces with the minimal length.

4.3.1. Hamilton-Jacobi Method in Deformed Spaces. In [28, 29], the classical limit of deformed spaces with the minimal length has been studied by replacing the quantum mechanical commutator by the Poisson bracket via

$$
\frac{1}{i \hbar}[\widehat{A}, \widehat{B}] \Longrightarrow\{A, B\} \text {. }
$$

Alternatively, we here use Hamilton-Jacobi method to probe the classical motion of a particle with the mass $m$ under the potential $V(x)$ in $1 D$ deformed spaces.

For the deformed commutation relation (64), the deformed time dependent Schrodinger equation is

$$
\begin{aligned}
& \frac{P^{2}((\hbar / i)(\partial / \partial x))}{2 m} \psi(x, t)+V(x) \psi(x, t) \\
& =i \hbar \frac{\partial \psi(x, t)}{\partial t} .
\end{aligned}
$$


Substituting the ansatz $\psi(x, t)=\exp [i S(x, t) / \hbar]$ into (108) and taking the limit $\hbar \rightarrow 0$, one finds that the leading order of (108) gives the classical Hamilton-Jacobi equation in deformed spaces

$$
\frac{P^{2}(\partial S / \partial x)}{2 m}+V(x)+\frac{\partial S}{\partial t}=0
$$

where $S(x, t)$ is the classical action. Since the potential $V(x)$ does not depend explicitly on time, we can separate the variables as

$$
S=W(x)-E t
$$

where $E$ can be identified with the total energy. Thus, the Hamilton-Jacobi equation becomes

$$
P\left(\frac{d W}{d x}\right)= \pm \sqrt{2 m[E-V(x)]} \equiv \pm P(x) .
$$

Defining $p(P)$ as an inverse function of $P(p)$, we obtain

$$
\frac{d W}{d x}= \pm p(P(x)) \equiv p(x) \text {. }
$$

Equation (112) can be integrated to

$$
W= \pm \int p(x) d x
$$

so that

$$
S= \pm \int p(x) d x-E t
$$

The solution for $x(t)$ comes from the transformation equation

$$
\begin{aligned}
C & =\frac{\partial S}{\partial E}= \pm \int \frac{\partial p(x)}{\partial E} d x-t \\
& = \pm \int \frac{m d x}{f(P(x)) P(x)}-t
\end{aligned}
$$

where the constant $C$ can be determined by the initial conditions and we use

$$
\begin{aligned}
\frac{\partial P(x)}{\partial E} & =\frac{m}{P(x)}, \\
\frac{d p}{d P} & =\frac{1}{f(P)} .
\end{aligned}
$$

Now we focus on the case with $f(P)=1+\beta P^{2}$.

First we consider the motion of a particle under the homogeneous field potential $V(x)=F x$. Equation (115) leads to

$$
\begin{aligned}
(1 & \left.-\frac{F}{E} x\right)\left[1-\frac{4 \beta m E}{3}\left(1-\frac{F}{E} x\right)\right] \\
& =\frac{F^{2} m}{2 E}\left(\frac{t+C}{m}\right)^{2},
\end{aligned}
$$

where we neglect the terms higher than $\mathcal{O}(\beta)$. Solving (117) for $x$ to $\mathcal{O}\left(\beta^{2}\right)$ gives

$$
x(t)=\frac{E}{F}-\frac{F}{2 m}(t+C)^{2}-m E \beta \frac{F^{3}}{3 E m^{2}}(t+C)^{4},
$$

which indicates that the initial conditions at $t=-C$ are $x(-C)=E / F \equiv x_{0}$ and $x^{\prime}(-C)=0$. Differentiating both sides of (117) twice with respect to $t$ gives the acceleration

$$
a \equiv \frac{d^{2} x}{d t^{2}}=-\frac{F}{m}\left[1+\frac{8}{3} \beta m^{2} x^{\prime 2}-\frac{8}{3} \beta m F\left(x-x_{0}\right)\right] .
$$

Using $x-x_{0}=m x^{\prime 2} / 2 F+\mathcal{O}(\beta)$, we find from (119) that

$$
a=-\frac{F}{m}\left(1+\frac{4}{3} \beta m^{2} x^{\prime 2}\right)+\mathcal{O}\left(\beta^{2}\right) .
$$

The equivalence principle is crucial in the foundations of general relativity. The weak equivalence principle is often referred to as the universality of free fall. A measure for the breakdown of the universality of free fall is the "Eotvos ratio" [30]:

$$
\eta(A, B)=\frac{2\left|a_{A}-a_{B}\right|}{\left|a_{A}+a_{B}\right|},
$$

which quantifies the normalized difference in the gravitational accelerations between two different bodies $A$ and $B$. In the modern torsion-balance experiment [31], the "Eotvos ratio" has been found to be

$$
\eta(\mathrm{Be}, \mathrm{Ti})=(0.3 \pm 1.8) \times 10^{-13}
$$

for the gravitational acceleration of Beryllium and Titanium towards the Earth. In the experiment of [31], they used

$$
\begin{aligned}
m_{\mathrm{Be}} & \approx m_{\mathrm{Ti}} \sim 1 \mathrm{~g}, \\
\left|m_{\mathrm{Be}}-m_{\mathrm{Ti}}\right| & \sim 1 \mu \mathrm{g} .
\end{aligned}
$$

Assuming that the gravitational and the inertial mass are the same, we obtain from (120) that

$$
\eta(A, B)=\frac{2\left|a_{A}-a_{B}\right|}{\left|a_{A}+a_{B}\right|} \sim \beta m_{A}\left|m_{A}-m_{B}\right| v^{2} .
$$

Plugging (123) into (124) gives

$$
\beta_{0}^{\mathrm{Be} / \mathrm{Ti}} \lesssim 1
$$

where we assume $v \sim 1 \mathrm{~m} / \mathrm{s}$. The superscript Be/Ti in the previous equation means that the upper bound $\beta_{0}$ is for the $\mathrm{Be} / \mathrm{Ti}$ test bodies. For quarks, it has been found that [29]

$$
\beta_{0}^{q} \approx 3^{2} N_{\text {nuc }}^{2} \beta_{0}^{\mathrm{Be} / \mathrm{Ti}}
$$

where $N_{\text {nuc }}$ is the number of nucleons in the test bodies. Since $N_{\text {nuc }} \sim 10^{-3} \mathrm{~kg} /\left(1.67 \times 10^{-27} \mathrm{~kg}\right)=10^{24}$, it is easy to see that

$$
\beta_{0}^{q} \lesssim 10^{49}
$$


which is much weaker than that from the precession of the perihelion of Mercury.

Now we will work out another simple example of a onedimensional harmonic oscillator with the potential

$$
V(x)=\frac{m \omega^{2} x^{2}}{2} .
$$

In this example, integrating (115) gives

$$
\begin{aligned}
\pm \omega(t+C)= & (1-\beta m E) \arcsin \left(\sqrt{\frac{m \omega^{2}}{2 E} x}\right) \\
& -\beta m E \sqrt{\frac{m \omega^{2}}{2 E}} x \sqrt{1-\frac{m \omega^{2} x^{2}}{2 E}},
\end{aligned}
$$

where the terms higher than $\mathcal{O}(\beta)$ are discarded. From the LHS of (129), one finds that the oscillator moves between $x= \pm \sqrt{2 E / m \omega^{2}}$, which is the same as in the usual case. Differentiating both sides of (129) with respect to $t$ gives the velocity

$$
\frac{d x}{d t}=\frac{ \pm \sqrt{2 E / m} \sqrt{1-m \omega^{2} x^{2} / 2 E}}{1+2 \beta m E\left(m \omega^{2} x^{2} / 2 E-1\right)},
$$

which implies that $\beta m E<1 / 2$ otherwise $d x / d t$ would blow up for some $x \in\left[-\sqrt{2 E / m \omega^{2}}, \sqrt{2 E / m \omega^{2}}\right]$. At $x=$ $\pm \sqrt{2 E / m \omega^{2}}$, we have $d x / d t=0$. Therefore, $x= \pm \sqrt{2 E / m \omega^{2}}$ are turning points and the motion is periodic. Furthermore, (129) gives that the period of the motion is

$$
T=\frac{2 \pi}{\omega}(1-\beta m E) .
$$

Since $\beta>0$, the effects of the minimal length would slow down the oscillation. Consider a simple gravity pendulum with the mass $m=0.1 \mathrm{~kg}$ and the length $l=1 \mathrm{~m}$, whose period in the usual case is $T_{0} \sim 2 \mathrm{~s}$. For the pendulum, the deformation parameter is

$$
\beta_{0}^{p} \approx \frac{\beta_{0}^{q}}{3^{2} N_{\text {nuc }}^{2}} \sim 10^{-53} \beta_{0}^{q} .
$$

The correction due to the minimal length to the period is

$$
|\Delta T|=T_{0} \beta^{p} m E \sim 10^{-55} \beta_{0}^{q} \mathrm{~s} \lesssim 10^{-19} \mathrm{~s}
$$

where we use $\beta_{0}^{q}<10^{36}$. The correction is too small to observe.

Generally, it can be inferred from the above two examples that the minimal length correction to some physical quantity $A$ of a nonrelativistic classical system is around

$$
\Delta_{A}=\frac{\left|A_{D}-A_{U}\right|}{\left|A_{U}\right|} \sim \beta m^{2} v^{2}
$$

where $A_{D(U)}$ is the value of $A$ which is calculated by the deformed theory (usual theory) and $m$ and $v$ are the typical mass and velocity of the test bodies, respectively. Since $\beta$ is for the test bodies, we have

$$
\beta \sim \beta_{0}^{q}\left(\frac{m}{\mathrm{~kg}}\right)^{-2} \times 10^{-54},
$$

where $\beta_{0}^{q}$ is for quarks. Define $\Delta_{A}^{E}=\left|A_{U}-A_{E}\right| /\left|A_{E}\right|$, where $A_{E}$ is the value of $A$ measured by the experiment. Thus, we find

$$
\begin{aligned}
& \Delta_{A} \lesssim \Delta_{A}^{E} \Longrightarrow \\
& \beta_{0}^{q} \lesssim\left(\frac{v}{\mathrm{~m}^{2} / \mathrm{s}^{2}}\right)^{-2} \Delta_{A}^{E} \times 10^{56} .
\end{aligned}
$$

For the precession of the perihelion of Mercury, $A$ is the angular velocity $\omega$ of Mercury, $\Delta_{\omega}^{E} \sim 10^{-11}$, and $v \sim 4 \times$ $10^{4} \mathrm{~m} / \mathrm{s}$. As a result, we can reproduce the upper bound on $\beta_{0}^{q}$ :

$$
\beta_{0}^{q} \lesssim 10^{36}
$$

It is also interesting to note that the upper bound on $\beta_{0}^{q}$ in (136) is proportional to $v^{-2}$ and independent of $m$. In order to put stronger constraints on $\beta_{0}^{q}$, one might need to look for the experiments with the high typical speed, possibly a relativistic one.

4.3.2. Statistical Physics in Deformed Spaces. In statistical physics we often need to calculate sums of the form

$$
\sum_{n} g\left(\frac{E_{n}}{k T}\right)
$$

where $E_{n}$ is the energy of $n$th level, $g$ is some function, and $k$ is the Boltzmann constant. For example, $g(x)=e^{-x}$ for the partition function of a quantum system obeying the Boltzmann statistics. Now we consider a $1 D$ nonrelativistic quantum system under the potential $V(x)$ in deformed spaces with the deformed commutation relation (64). For this system, we will use the Bohr-Sommerfeld quantization condition (81) to show that, under the condition $E_{n+1}-E_{n} \ll$ $k T$, the sum in (138) is equal to

$$
\sum_{n} g\left(\frac{E_{n}}{k T}\right) \approx \int \frac{d x d P}{2 \pi \hbar f(P)} g\left(\frac{E(P, x)}{k T}\right),
$$

where $E(P, x)=P^{2} / 2 m+V(x)$.

First, we observe that the integral in (81) is exactly half the area surrounded by the classical trajectory of the particle in phase space of $p$ and $x$. Thus, we find

$$
A_{E_{n}}=(2 n+1) \pi \hbar,
$$

where $E_{n}$ is the energy of $n$th level and $A_{E_{n}}$ denote the entire area inside the trajectory corresponding to the energy $E_{n}$. Let us denote the domain enclosed between $n$th and $n+1$ th trajectory as $D_{n}$, whose area is $A_{E_{n+1}}-A_{E_{n}}=2 \pi \hbar$. Thus, we have

$$
\int_{D_{n}} d x d p=2 \pi \hbar
$$


It is easy to see that

$$
g\left(\frac{E_{n}}{k T}\right)=g\left(\frac{E_{n}}{k T}\right) \int_{D_{n}} \frac{d x d p}{2 \pi \hbar} .
$$

If $E_{n+1}-E_{n} \ll k T$, we find

$$
g\left(\frac{E_{n}}{k T}\right) \approx \int_{D_{n}} \frac{d x d p}{2 \pi \hbar} g\left(\frac{E(P, x)}{k T}\right) .
$$

The sum in (138) becomes

$$
\begin{aligned}
\sum_{n} g\left(\frac{E_{n}}{k T}\right) & \approx \sum_{n} \int_{D_{n}} \frac{d x d p}{2 \pi \hbar} g\left(\frac{E(P, x)}{k T}\right) \\
& =\int \frac{d x d p}{2 \pi \hbar} g\left(\frac{E(P, x)}{k T}\right),
\end{aligned}
$$

where the integral is over the entire phase space of $x$ and $p$. Using $d P(p) / d p=f(P)$, we conclude the proof of (139). Note that formula (139) has also been obtained in [32] by calculating the Jacobian $J=\partial(X, P) / \partial(x, p)$ and in $[33,34]$ by using Liouville theorem.

Now consider a $1 D$ harmonic oscillator with the potential $V(x)=m \omega^{2} x^{2} / 2$. For $T \gg\left(E_{n+1}-E_{n}\right) / k \sim \hbar \omega / k$, the partition function for the oscillator is

$$
\begin{aligned}
Z & =\int \frac{d x d P}{2 \pi \hbar f(P)} \exp \left[-\frac{1}{2 k T}\left(\frac{P^{2}}{m}+m \omega^{2} x^{2}\right)\right] \\
& =\frac{k T}{\hbar \omega \sqrt{\pi}} \int_{-\infty}^{\infty} \frac{d x}{f(\sqrt{2 k T m x})} \exp \left(-x^{2}\right),
\end{aligned}
$$

where $x=P / \sqrt{2 k T m}$. Suppose $f(P)=1+\beta P^{2}$. The partition function becomes

$$
Z=\frac{k T}{\hbar \omega \sqrt{\pi}} \int_{-\infty}^{\infty} \frac{d x}{1+2 k T m \beta x^{2}} \exp \left(-x^{2}\right) .
$$

If $k \operatorname{Tm} \beta \ll 1$, we find

$$
Z \approx \frac{k T}{\hbar \omega}(1-k T m \beta) .
$$

If $k \operatorname{Tm} \beta \gg 1$, we find

$$
\begin{aligned}
Z & =\frac{k T}{\hbar \omega \sqrt{\pi}} \frac{1}{\sqrt{2 k T m \beta}} \int_{-\infty}^{\infty} \frac{d y}{1+y^{2}} \exp \left(-\frac{y^{2}}{2 k T m \beta}\right) \\
& \approx \frac{\sqrt{k T \pi}}{\sqrt{2 m \beta} \hbar \omega},
\end{aligned}
$$

where $y=\sqrt{2 k \operatorname{Tm} \beta} x$. The average energy of the oscillator is

$$
\begin{aligned}
\bar{E} & =-\frac{\partial}{\partial(1 / k T)} \ln Z \\
& \approx \begin{cases}k T(1-\beta m k T) & \text { for } k T m \beta \ll 1 \\
\frac{1}{2} k T & \text { for } k T m \beta \gg 1 .\end{cases}
\end{aligned}
$$

If $k \operatorname{Tm} \beta \gg 1$, the energy for one degree of freedom in the equipartition theorem in deformed spaces is only half of that in the usual case.

Einstein assumed that the atoms in a crystal are equivalent to $3 N$ harmonic oscillators and calculated heat capacities of solids. For an atom with the standard atomic weight $A_{r}$, we find

$$
\frac{1}{k m \beta^{a}} \sim 10^{18}\left(\frac{A_{r}}{100}\right)\left(\frac{10^{36}}{\beta_{0}^{q}}\right) \mathrm{K} .
$$

Usually heat capacities of solids are measured at $T \sim 10^{2} \mathrm{~K}$. In this case, (149) gives that the molar specific heat of a solid for $\hbar \omega / k \ll T \ll 1 / k m \beta^{a}$ is

$$
C=3 R\left(1-\beta^{a} m k T\right)
$$

where $R=8.31 \mathrm{JK}^{-1} \mathrm{~mol}^{-1}$ is the gas constant. For a solid consisting of atoms with $A_{r}$, we have

$$
\Delta C=\left|\frac{C-3 R}{3 R}\right| \sim 10^{-52} \beta_{0}^{q}\left(\frac{100}{A_{r}}\right)\left(\frac{T}{100 \mathrm{~K}}\right) .
$$

For example, the heat capacity of Tungsten at $T=400 \mathrm{~K}$ is $C=24.92 \mathrm{JK}^{-1} \mathrm{~mol}^{-1}[35]$, which implies

$$
\beta_{0}^{q} \lesssim 10^{50}
$$

\section{Conclusions}

In this paper, we considered a homogeneous field in the deformed quantum mechanics with minimal length. The physical motivation for this is to obtain the WKB connection formula and prove the Bohr-Sommerfeld quantization rule rigorously in the deformed quantum mechanics. By studying the leading asymptotic behavior of the physically acceptable wave function in the physical region, we found the contour for its integral representation. Through the integral representation, the asymptotic expansions of the physically acceptable wave function at both large positive and large negative values of $\rho$ were given.

We then used the obtained asymptotic expansions to get the WKB connection formula, which proceeds from classically forbidden region to classically allowed one through a smooth turning point, and had the Bohr-Sommerfeld quantization rule proved rigorously up to $\mathcal{O}\left(\beta^{2}\right)$. A new interesting feature appearing in the presence of deformation was that our WKB connection formula does not work for a sharp turning point. The connection through such a point might need a higher order approximation to the potential near it.

We discussed the competition between the quantum gravity correction on the first-order WKB approximation and the second-order WKB approximation. If the former is not overwhelmed by the latter, the energy of the particle considered should be high enough according to (90). We also showed that if the energy levels $E_{n}^{(0)}$ of a bound state are given in the ordinary quantum mechanics, the deformed energy levels are

$$
E_{n}=E_{n}^{(0)}+\beta E_{n, 0}^{1}+\mathcal{O}(\hbar) \mathcal{O}(\beta)+\mathcal{O}\left(\beta^{2}\right),
$$


where $\beta E_{n, 0}^{1}$ is the $\mathcal{O}(\beta)$ quantum gravity correction on the first-order WKB approximation. Finally, we used the Hamilton-Jacobi method to study the effects of the minimal length on the classical motions. The Bohr-Sommerfeld quantization was then used to investigate statistical physics in deformed spaces with the minimal length. Upper bounds on $\beta_{0}^{q}$ were obtained from measurements of the "Eotvos ratio" and the heat capacity of Tungsten.

\section{Conflict of Interests}

The authors declare that there is no conflict of interests regarding the publication of this paper.

\section{Acknowledgments}

The authors are grateful to Benrong $\mathrm{Mu}$, Houwen $\mathrm{Wu}$, and Zheng Sun for useful discussions. This work is supported in part by NSFC (Grant nos. 11175039, 11147106, 11005016, and 11375121) and the Fundamental Research Funds for the Central Universities.

\section{References}

[1] G. Veneziano, "A stringy nature needs just two constants," Europhysics Letters, vol. 2, no. 3, pp. 199-204, 1986.

[2] D. J. Gross and P. F. Mende, "String theory beyond the Planck scale," Nuclear Physics B, vol. 303, no. 3, pp. 407-454, 1988.

[3] D. Amati, M. Ciafaloni, and G. Veneziano, "Can space-time be probed below the string size?” Physics Letters B, vol. 216, no. 1-2, pp. 41-47, 1989.

[4] L. J. Garay, “Quantum gravity and minimum length," International Journal of Modern Physics A, vol. 10, no. 2, pp. 145-165, 1995.

[5] M. Maggiore, "The algebraic structure of the generalized uncertainty principle," Physics Letters B, vol. 319, no. 1-3, pp. 83-86, 1993.

[6] A. Kempf, G. Mangano, and R. B. Mann, "Hilbert space representation of the minimal length uncertainty relation," Physical Review. D. Third Series, vol. 52, no. 2, article 1108, 1995.

[7] S. Hossenfelder, "Minimal length scale scenarios for quantum gravity," Living Reviews in Relativity, vol. 16, no. 2, 2013.

[8] S. Hossenfelder, M. Bleicher, S. Hofmann, J. Ruppert, S. Scherer, and H. Stöcker, "Signatures in the Planck regime," Physics Letters Section B, vol. 575, no. 1-2, pp. 85-99, 2003.

[9] A. Camacho, "Generalized uncertainty principle and quantum electrodynamics," General Relativity and Gravitation, vol. 35, no. 7, pp. 1153-1160, 2003.

[10] M. Maziashvili, "Implications of minimum-length deformed quantum mechanics for QFT/QG," Fortschritte der Physik, vol. 61, no. 6, pp. 685-700, 2013.

[11] M. S. Berger and M. Maziashvili, "Free particle wave function in light of the minimum-length deformed quantum mechanics and some of its phenomenological implications," Physical Review D, vol. 84, no. 4, Article ID 044043, 2011.

[12] S. Das and E. C. Vagenas, "Universality of quantum gravity corrections," Physical Review Letters, vol. 101, no. 22, Article ID 221301, 2008.

[13] L. N. Chang, D. Minic, N. Okamura, and T. Takeuchi, "Exact solution of the harmonic oscillator in arbitrary dimensions with minimal length uncertainty relations," Physical Review D, vol. 65, no. 12, Article ID 125027, 2002.

[14] R. Akhoury and Y.-P. Yao, "Minimal length uncertainty relation and the hydrogen spectrum," Physics Letters B, vol. 572, no. 1-2, pp. 37-42, 2003.

[15] T. V. Fityo, I. O. Vakarchuk, and V. M. Tkachuk, "Onedimensional Coulomb-like problem in deformed space with minimal length," Journal of Physics A: Mathematical and General, vol. 39, no. 9, pp. 2143-2149, 2006.

[16] F. Brau and F. Buisseret, "Minimal length uncertainty relation and gravitational quantum well," Physical Review D, vol. 74, no. 3, Article ID 036002, 2006.

[17] P. Pedram, K. Nozari, and S. H. Taheri, "The effects of minimal length and maximal momentum on the transition rate of ultra cold neutrons in gravitational field," Journal of High Energy Physics, vol. 2011, article 93, 2011.

[18] P. Pedram, "Minimal length and the quantum bouncer: a nonperturbative study," International Journal of Theoretical Physics, vol. 51, no. 6, pp. 1901-1910, 2012.

[19] A. F. Ali, S. Das, and E. C. Vagenas, "Discreteness of space from the generalized uncertainty principle," Physics Letters. B, vol. 678, no. 5, pp. 497-499, 2009.

[20] A. F. Ali, S. Das, and E. C. Vagenas, "Proposal for testing quantum gravity in the lab," Physical Review D, vol. 84, no. 4, Article ID 044013, 2011.

[21] P. Pedram, "New approach to nonperturbative quantum mechanics with minimal length uncertainty," Physical Review D, vol. 85, no. 2, Article ID 024016, 2012.

[22] T. V. Fityo, I. O. Vakarchuk, and V. M. Tkachuk, "WKB approximation in deformed space with minimal length," Journal of Physics. A. Mathematical and General, vol. 39, no. 2, article 379, 2006.

[23] P. Pedram, "A higher order GUP with minimal length uncertainty and maximal momentum," Physics Letters B, vol. 714, no. 2-5, pp. 317-323, 2012.

[24] C.-L. Ching, R. R. Parwani, and K. Singh, "Constraints and spectra of a deformed quantum mechanics," Physical Review D, vol. 86, no. 8, Article ID 084053, 2012.

[25] C. M. Bender and S. A. Orszag, Applied Mathematical Methods for Scientists and Engineers, McGraw-Hill, 1978.

[26] C. L. Ching and R. Parwani, "Scattering and bound states of adeformed quantum mechanics," http://arxiv.org/abs/1207.1519.

[27] L. Landau and E. Lifshitz, Quantum Mechanics, Pergamon Press, Oxford, UK, 2000.

[28] S. Benczik, L. N. Chang, D. Minic, N. Okamura, S. Rayyan, and T. Takeuchi, "Short distance versus long distance physics: the classical limit of the minimal length uncertainty relation," Physical Review D, vol. 66, no. 2, Article ID 026003, 2002.

[29] C. Quesne and V. M. Tkachuk, "Composite system in deformed space with minimal length," Physical Review A, vol. 81, no. 1, Article ID 012106, 2010.

[30] C. M. Will, "The confrontation between general relativity and experiment," Living Reviews in Relativity, vol. 17, no. 4, 2014.

[31] S. Schlamminger, K.-Y. Choi, T. A. Wagner, J. H. Gundlach, and E. G. Adelberger, "Test of the equivalence principle using a rotating torsion balance," Physical Review Letters, vol. 100, no. 4, Article ID 041101, 2008.

[32] T. V. Fityo, "Statistical physics in deformed spaces with minimal length," Physics Letters A, vol. 372, no. 37, pp. 5872-5877, 2008. 
[33] P. Wang, H. Yang, and X. Zhang, "Quantum gravity effects on statistics and compact star configurations," Journal of High Energy Physics, vol. 2010, article 43, 2010.

[34] L. N. Chang, D. Minic, N. Okamura, and T. Takeuchi, "The effect of the minimal length uncertainty relation on the density of states and the cosmological constant problem," Physical Review D, vol. 65, Article ID 125028, 2002.

[35] D. R. Lide, Ed., CRC Handbook of Chemistry and Physics, Internet Version 2005, CRC Press, Boca Raton, Fla, USA, 2005, http://www.hbcpnetbase.com/. 

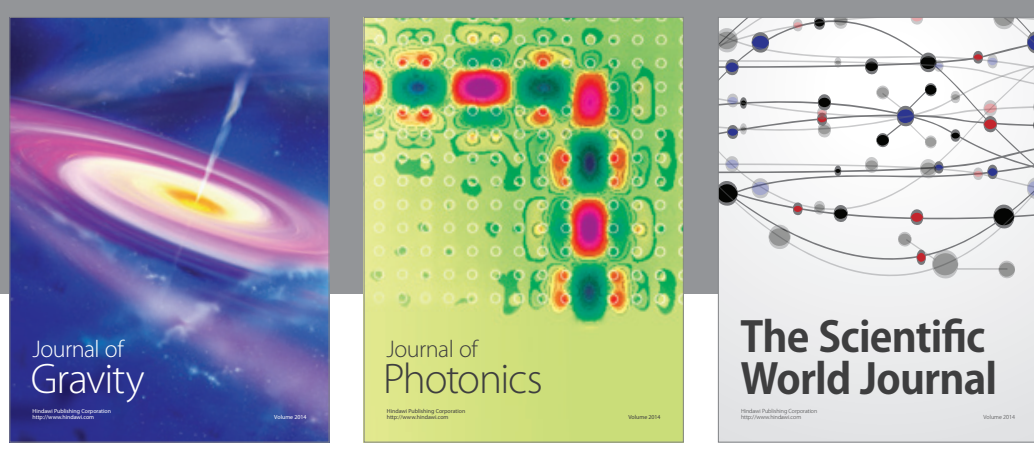

The Scientific World Journal
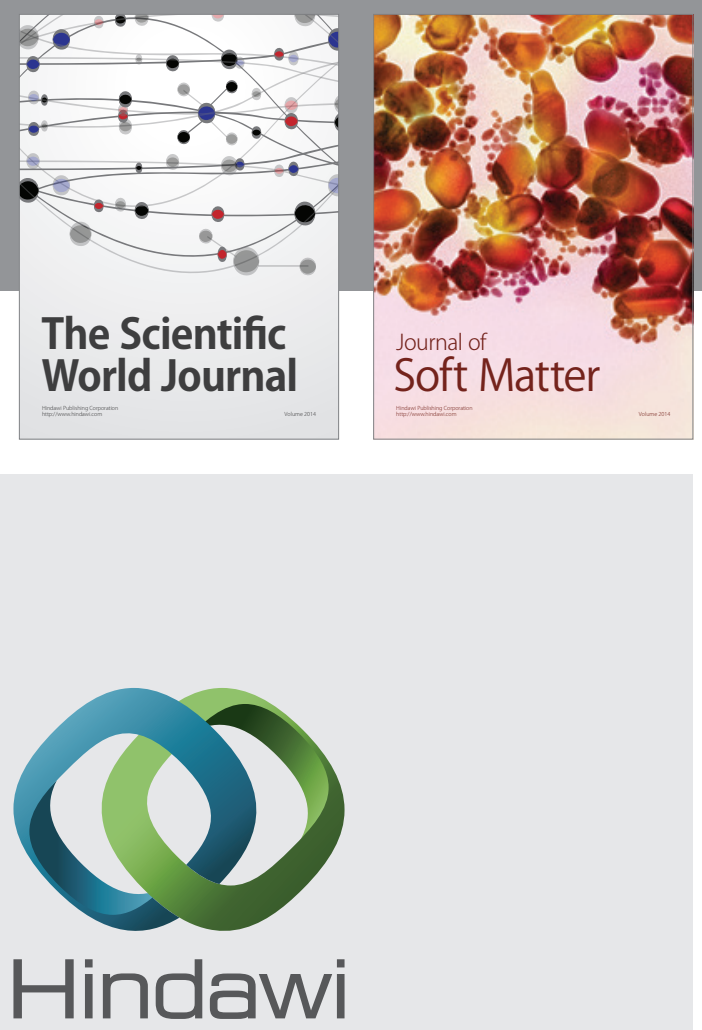

Submit your manuscripts at

http://www.hindawi.com

nternational Journal of

Statistical Mechanics
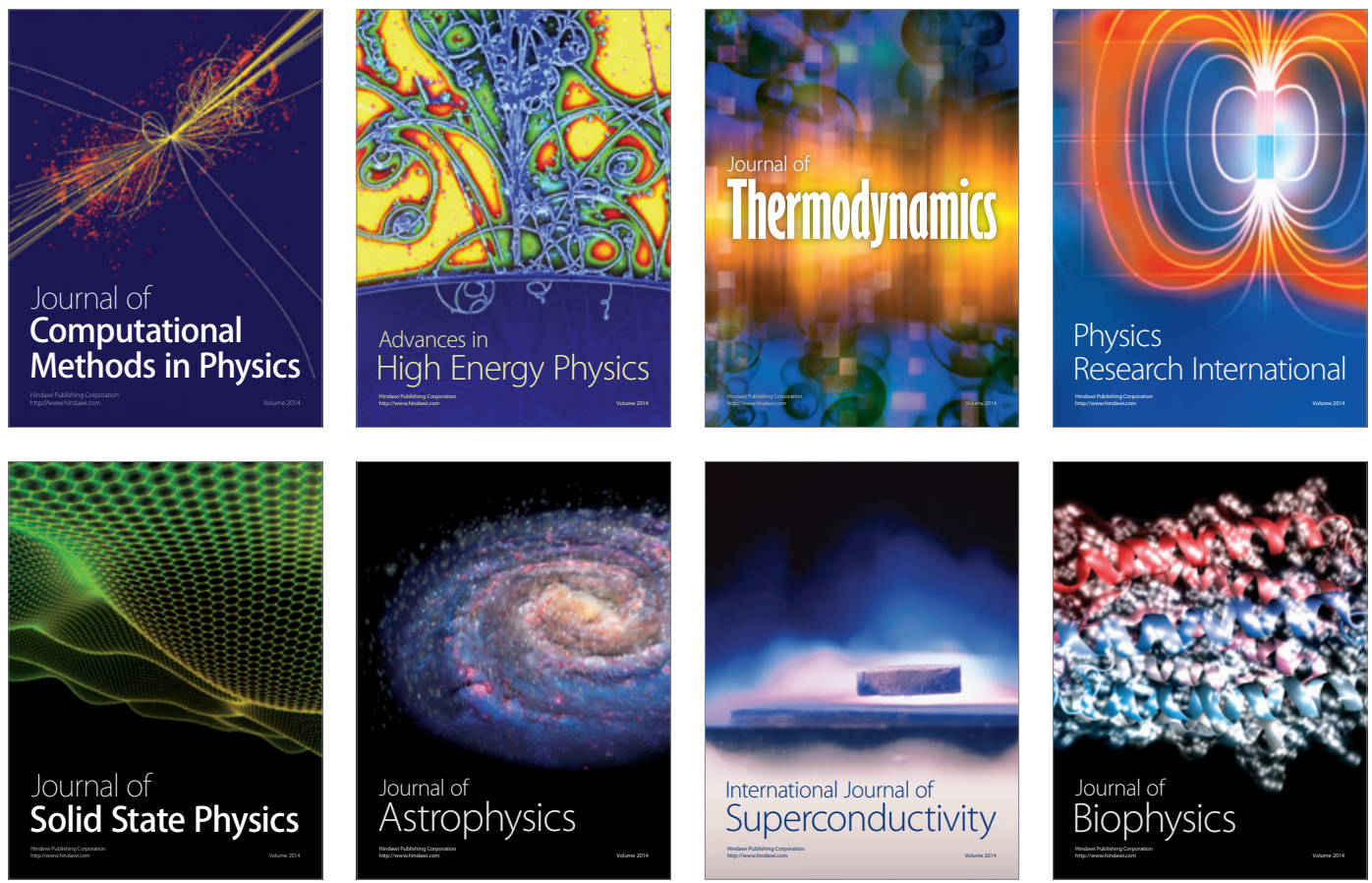
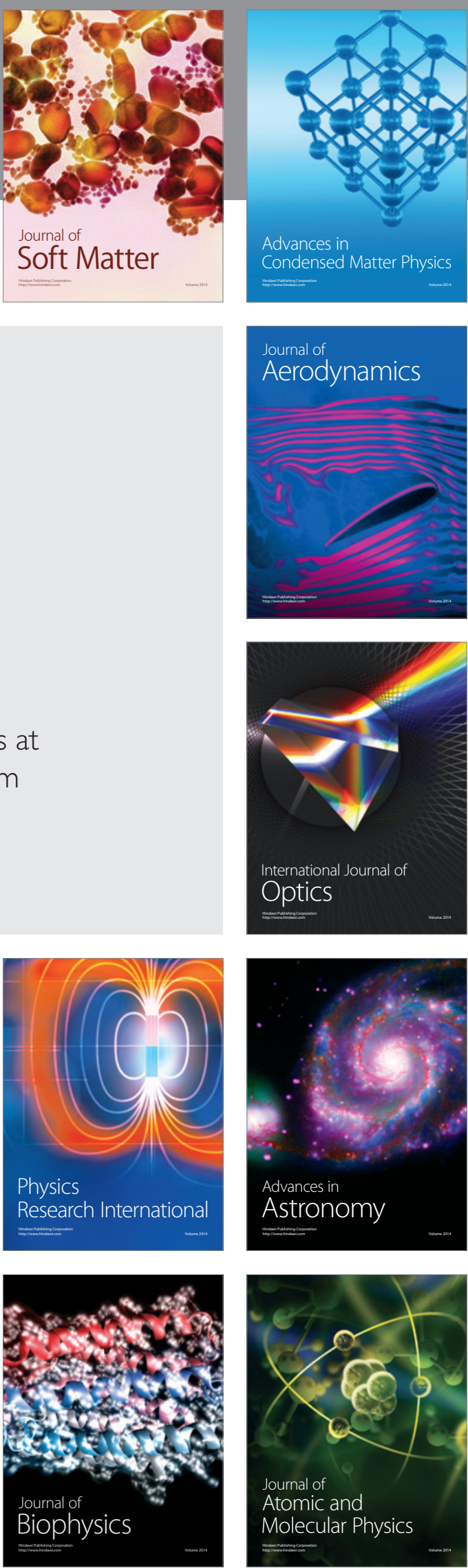